\title{
Design and Implementation of Intelligent Insulation Monitoring System for Ship Grid Based on ProfiNet
}

\author{
Jinlong Sun ${ }^{1, a}$, Zhiliang $\mathrm{Wu}^{2, \mathrm{~b}}$ \\ ${ }^{1}$ Marine Engineering College, Dalian Maritime University, Dalian 112026, China \\ ${ }^{2}$ Marine Engineering College, Dalian Maritime University, Dalian 112026, China \\ aemail: xcxsjl@sina.com, bemail: wzldmu@126.com
}

Key words: ship grid; insulation monitoring; ProfiNet; S7-1200 PLC; HMI

\begin{abstract}
According to the requirements of remote insulation monitoring for automated marine power station, the intelligent insulation monitoring system for ship grid was designed. On the basis of ProfiNet, a new S7-1200 PLC with ProfiNet interface of Siemens is chosen as the core controller by the system, making MT8100i HMI into industrial Ethernet, so that the design of monitoring system was completed. Compared with the traditional megohmmeter, the system has distinct advantages in monitoring accuracy, communication rate and distance, and anti-interference. Besides, the system can realize not only remote real-time insulation monitoring but also fault location.
\end{abstract}

\section{Introduction}

With the rapid development of computer control technology, the trend of ship automation and large-scale and the requirements of continuity of power supply for marine power system keep on increasing. The electric fire accidents caused by the power grid insulation failure are a dime a dozen. So real-time monitoring ship grid insulation to the ground is of great significance for the safe navigation $^{[1]}$.

At present, the traditional dial-type mega-ohm meter usually adopted on ship has been unable to meet the requirements of the modern marine power station automation in terms of remote information interaction and intelligentialize, and it gradually being replaced by the new type digital mega-ohm meter, therefore the research and development of new type intelligent insulation monitoring system has a high practical value. In the system, S7-1200 and HMI are configurated to a network by industrial Ethernet based on ProfiNet to monitor the insulation condition of marine power grid remotely and timely, which can be send to HMI by Ethernet to display, alarm audibly and visually, record historical date, and locate fault phase.

\section{Insulation monitoring methods for ship grid}

There are some insulation monitoring methods for ship such as DC signal monitoring, dual-frequency signal monitoring, zero-sequence current monitoring and so on ${ }^{[2]}$.

The most common method of real-time monitoring grid insulation to the ground is megohmmeter method, known as DC signal monitoring method. The essence of DC signal monitoring method is to input a DC signal into marine power grid, and the DC signal in the circuit will fluctuate as the change of insulation resistance, therefore ground insulation can be displayed on the meter through a series of transformations ${ }^{[3]}$.

The equivalent schematic diagram of DC signal monitoring method is shown in Fig1. The DC signal provided by a DC power source flows into a circuit formed by the current limiting resistor R, internal resistance of meter $R_{R}$, the earth, the equivalent resistor $R_{X}$ and the stator winding, to measure ship grid insulation resistance to the ground. According to Fig. 1, the current flowing into the meter is

$$
R_{X}=\frac{U}{I}-R-R_{R}
$$




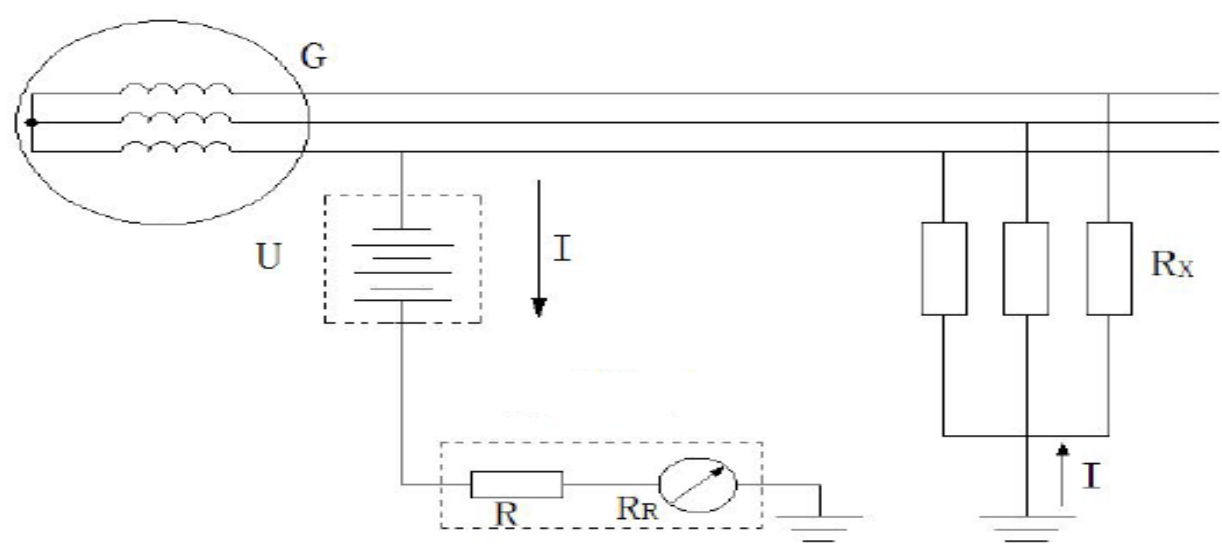

Fig. 1 the equivalent schematic diagram of DC signal method

The current flowing through the meter I changes with the resistor $R_{X}$, because the supply voltage $\mathrm{U}$, the current limiting resistor $\mathrm{R}$ and the internal resistance of meter $\mathrm{R}_{\mathrm{R}}$ are constant. Consequently, The current flowing through the meter I can exactly reflect the ship grid insulation to the ground.

Compared with other methods, DC signal monitoring method is more common in the field of ship. However, this method can only determine whether the grid insulation fault exists , but can't locate the fault phase exactly. For now, insulation indicating light method is usually adopted to locate which phase occurred insulation failure ${ }^{[4]}$.

\section{The hardware design of the intelligent insulation monitoring system}

The voltage signal is obtained from ship grid by means of adopting DC signal monitoring method, input to PLC through rectifier and filter circuit, and transmitted to HMI via ProfiNet interface to display, record and alarm response in the system. WEINVIEW MT8100i touch screen is utilized as the upper computer, configured by EasyBuilder8000 software. SIEMENS S7-1200 PLC is chosen as the core controller, programmed by TIA Portal V13. The structure diagram is shown in fig. 2.

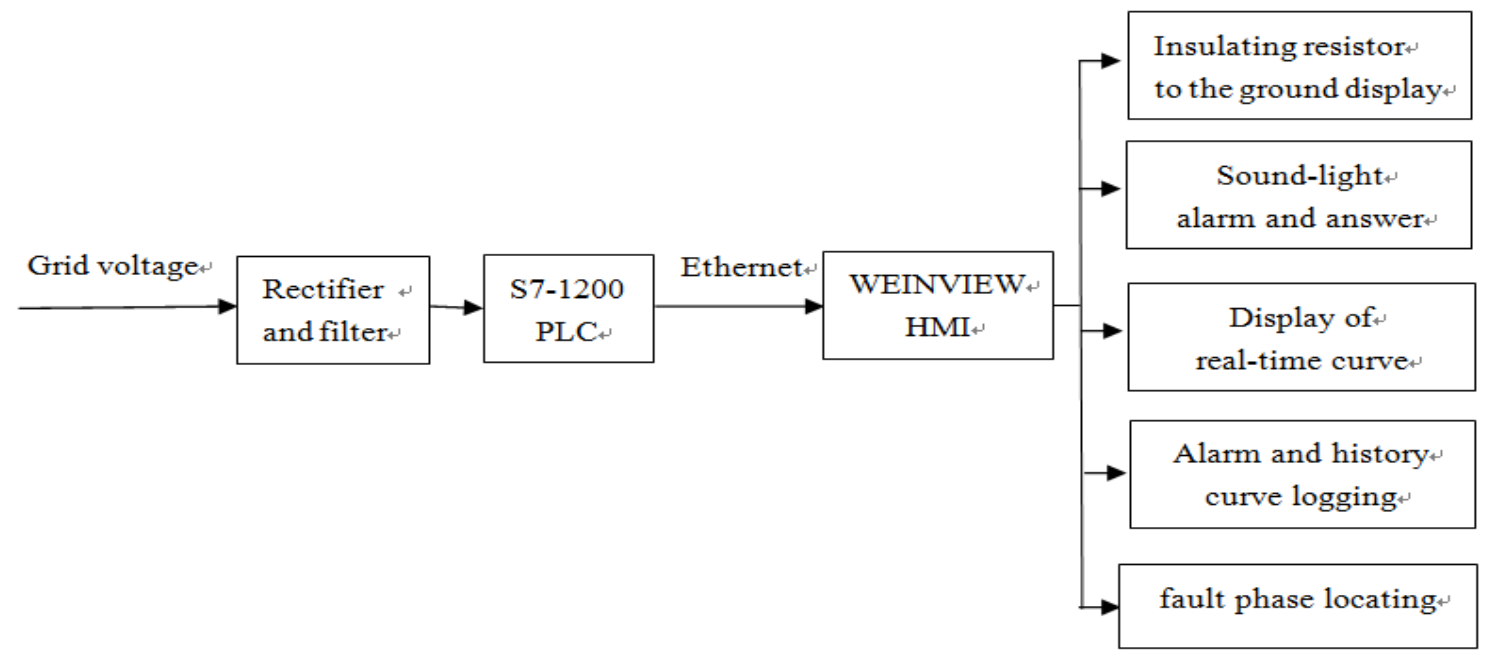

Fig. 2 The structure diagram of the insulation monitoring system

\subsection{Core controller and upper computer}

PLC is a kind of computer system designed for the area of industrial control specially ${ }^{[5]}$. SIEMENS S7-1200 PLC is chosen as the core controller by the system, which can communicate with programmable device and HMI remotely via Ethernet through CPU module with ProfiNet interface. The analog module SM1234 acquire voltage signal from ship grid through rectifier and filter, and then the CPU module process the date, ultimately, the date being transmitted to HMI to display.

The system chose WEINVIEW MT8100i touch screen with ProfiNet interface as the upper computer, so it can be connected to Ethernet, meeting the requirements of remote monitoring. 


\subsection{The circuit of collecting signal}

The system senses the insulation condition of the grid by collecting the variation of signal, so it needs no high voltage but the requirements of analog input.

The $15 \mathrm{~V}$ ac signal can be acquired through two transformation, and then the signal is rectified, filtered and stabilized in stabilivolt LM7812, to get a constant $12 \mathrm{~V}$ dc voltage signal. The dc signal reachs the grid from the positive of LM7812 via the switch $K$, to earth through the insulation resistance $R_{X}$, and then flows into the negative terminals via sampling resistance $R_{1}$ and resistor divider $\mathrm{R}_{2}$ and forming into a circuit loop. The electrical signal sampled by resistor $\mathrm{R}_{1}$ is input into analog module SM1234. The specific circuit figure is shown in fig. 3.

On the premise that the sampling voltage doesn't exceed the analog input range, try to ensure that the range of $U_{1}$ is larger, when $R_{X}$ varies between 0 and $10 \mathrm{M} \Omega$, and the larger $R_{X}$ is allowable for analog input range.

The input voltage range of analog port is $-10 \mathrm{~V} \sim 10 \mathrm{~V}$, so the voltage across sampling resistor $\mathrm{R}_{1}$ shouldn't exceed $10 \mathrm{~V}$.

The voltage across the resistor $R_{1}$ is the largest, when grid grounding occurs, namely $R_{x}=0$.

$$
\mathrm{U}_{1}+\mathrm{U}_{2}=\mathrm{U}
$$

In the formula: $\mathrm{U}_{1}$ is the voltage across the sampling resistor $\mathrm{R}_{1}$;

$\mathrm{U}_{2}$ is the voltage across the resistor divider $\mathrm{R}_{2}$;

$\mathrm{U}$ is the voltage of stabilivolt LM7812, namely $12 \mathrm{~V}$.

When $U_{1}=10 \mathrm{~V}, \mathrm{U}_{2}=2 \mathrm{~V}$, because $\mathrm{U}=12 \mathrm{~V}$. So $\mathrm{U}_{1} / \mathrm{U}_{2}=5$, deducing $\mathrm{R}_{1} / \mathrm{R}_{2}=5$. According to $\mathrm{R}_{1} / \mathrm{R}_{2}=5$, $\mathrm{U}_{1}$ tops its ceiling of $10 \mathrm{~V}$. The lower limit of $\mathrm{U}_{1}$ can be obtained as follwing.

The computing formula of sampling resistor can be listed as the following:

$$
U_{1}=\frac{U}{R_{1}+R_{2}+R_{X}} R_{1}
$$

The formula (3) can be obtained by (2):

$$
U_{1}=\frac{U}{1+\frac{R_{2}}{\boldsymbol{R}_{1}}+\frac{\boldsymbol{R}_{X}}{\boldsymbol{R}_{1}}}
$$

Because $U=12 \mathrm{~V}$, and $R 2 / R 1=0.2$, so $U_{1}$ arrives lower limit, when $R_{X}$ is infinite. Let $R_{1}=5 M \Omega$, $R_{2}=1 M \Omega$, so when $R_{X}=10 M \Omega, U_{1}=3.75 V$; when $R_{X}$ is infinite, $U_{1}=0$.

The voltage across the sampling resistor $U_{1}$ varies between $0 \mathrm{~V}$ and $10 \mathrm{~V}$, when insulation resistance $R_{X}$ varies between $0 M \Omega$ and infinite. The requirements of analog input can be met, and the variation range is large.

The formula (4) can be obtained by (3):

$$
R_{X}=\frac{U R_{1}}{U_{1}}-R_{1}-R_{2}
$$

The resistance of $R_{x}$ can be calculated because $R 1=5 M \Omega, R 2=1 M \Omega, U=12 V$, and $U_{1}$ is obtained by PLC, and $\mathrm{R}_{\mathrm{X}}$ is transmitted to upper computer via Ethernet.

The system requires that the alarm signal is sent when insulation resistor $R_{x}$ is below $1 M \Omega$, and the alarm limit can be adjusted in the PLC program. According to the formula (3), when $R_{X}=1 M \Omega$, $\mathrm{U}_{1}$ equals 8.75. The alarm signal is generated, when $\mathrm{U}_{1}$ collected by PLC is larger than $8.75 \mathrm{~V}$. 


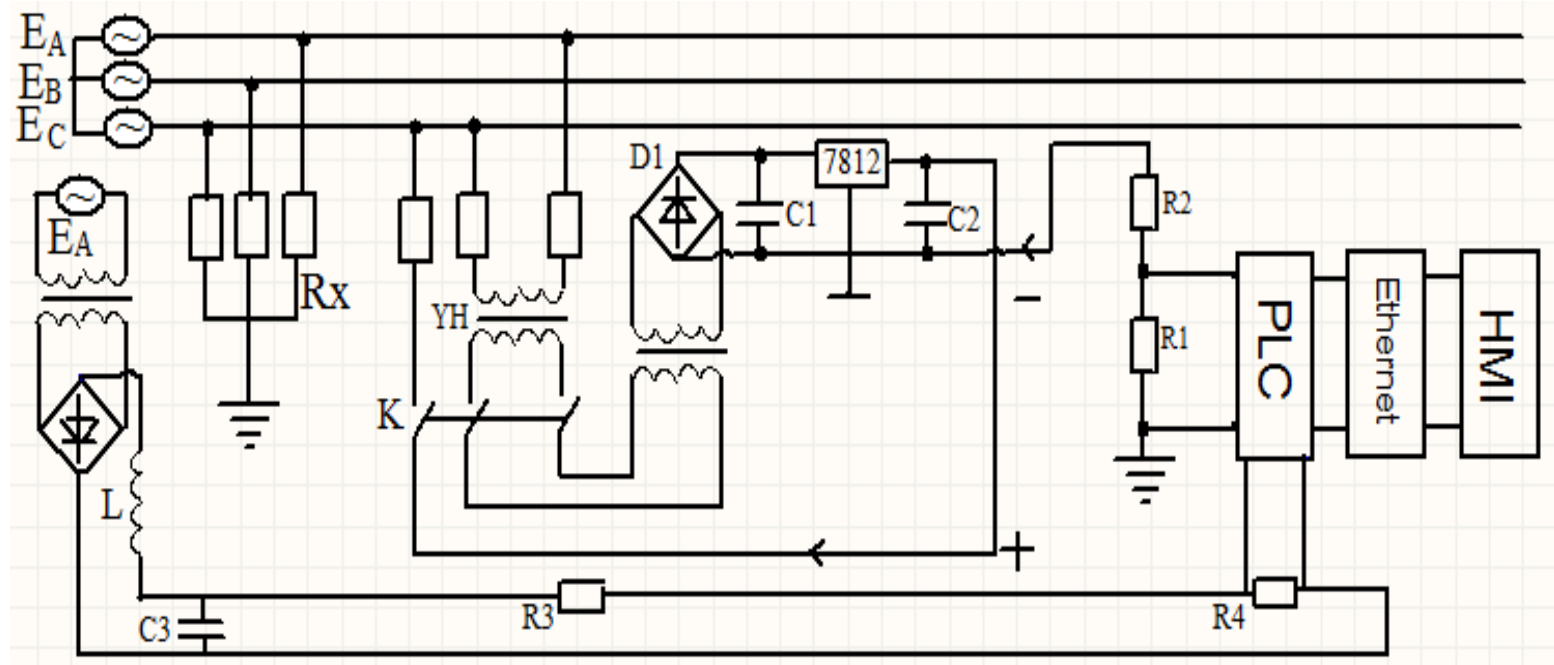

Fig. 3 The diagram of signal gather circuit

Compared with the traditional megohmmeter, the system can realize not only remote real-time insulation monitoring but also fault location.

Taking phase A for example, $15 \mathrm{v}$ ac signal can be acquired through transformation for phase voltage, as shown in fig. 3 . The ac signal is input single phase bridges rectifying circuit, and filtered by inductor $L$ and capacitor $C_{3}$. When phase voltage $E_{A}$ is normal, the voltage across the resistor $R_{3}$ and $\mathrm{R}_{4}$ is $12 \mathrm{~V}$, namely

$$
\mathrm{U}_{3}+\mathrm{U}_{4}=12 \mathrm{~V}
$$

In the formula: $\quad \mathrm{U}_{3}$ is the voltage across the resistor divider $\mathrm{R}_{3}$;

$\mathrm{U}_{4}$ is the voltage across the sampling resistor $\mathrm{R}_{4}$.

In order to ensure that the voltage across resistor $\mathrm{R}_{4}$ meets the requirements of PLC analog input, let $\mathrm{R}_{3}=\mathrm{R}_{4}=5 \mathrm{M} \Omega$, so the voltage across the sampling resistor $\mathrm{U}_{4}$ varies between $0 \mathrm{~V}$ and $6 \mathrm{~V}$, when the phase voltage varies between $0 \mathrm{~V}$ and $220 \mathrm{~V}$, so the requirements of analog input can be met absolutely.

The fault phase limit value of alarming is set to $5.8 \mathrm{~V}$, to prevent the error alarm caused by voltage fluctuations, so when $\mathrm{U} 4 \leq 5.8 \mathrm{~V}$, and $\mathrm{U} 1 \geq 8.75 \mathrm{~V}$, the contact of PLC related to fault phase is set to 1 , generating alarm signal and displaying the alarm in the touch screen.

\section{The software design of the intelligent insulation monitoring system}

\subsection{The program design for S7-1200PLC}

S7-1200PLC adopts the TIA Portal V13 software to accomplish hardware configuration and program design. Graphical configuration and diagnosis is allowable in the software, adopting structured programming, so the efficiency of programming is improved sharply ${ }^{[6]}$. PLC collects analog signal and processes data as the core controller, which transfers digital signal to upper computer to display. The data collection and phase A alarm program of PLC is shown in fig. 4, first of all, the voltage signal collected is normalized, and then, compared with alarming limit value, finally alarm is realized.

The system can realize not only remote real-time insulation monitoring but also fault location. Three phases voltage are obtained three single phase bridges rectifying circuit, and the rectified dc signal is input into PLC to process. When the phase voltage isn't normal and $U 1 \geq 8.75$, the contact of PLC related to fault phase is set to 1, transmitting to upper computer via Ethernet to display and alarm audibly and visually. To response the sound-light alarm, answering key is set in the touch screen. 


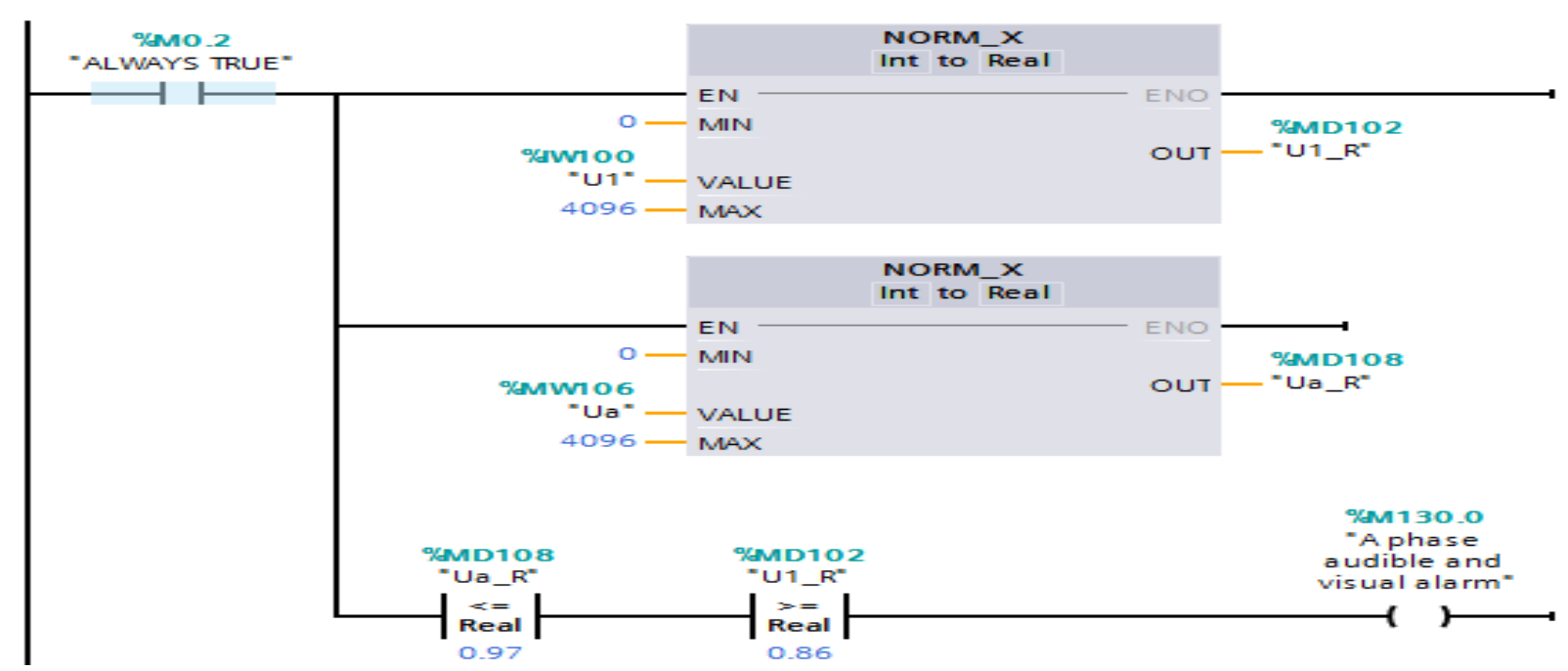

\subsection{The device configuration of touch screen HMI}

Fig.4 The data collection and phase A alarm program of PLC

In this design, the interface adopts the product which compiled by the configuring software EB800 project manager (PC port) that belongs to WEINVIEW company. This software is a appropriative for the touch screen which produced by WEINVIEW company, it can be compatible with other automation equipments, and it is a highly open and process-visible system ${ }^{[7]}$. There are three user windows built in this design, they are online insulation monitoring window, history curve window and alert data recording window, realizing all requirements for controlling, displaying, alerting and history recording. The history curve window and alert data recording window are designed mainly for users to look up and analyze history data, to predict the insulation of power grid. A standard $3 \mathrm{M} \Omega$ resistor is connecting power grid and the ground for testing the performance of the device, the indicating data of megohmmeter and the designed interface are shown as fig.5, the precision of the design is much higher than the traditional megohmmeter.

\section{Conclusion}

The intelligent insulation monitoring system adopts ProfiNet Ethernet communications technology, so it can implement long-range monitoring. Through repeated experiments on marine power station, its results demonstrate that the system meets requirements of remote insulation monitoring for automated marine power station, because of long-range monitoring, fault phase locating and high degree of accuracy. Compared with the traditional insulation monitoring device, the system has obvious advantages, which has played a certain reference function for the development of monitoring system for ship power station.

\section{References}

[1]Cao Jian, Guo Jianming, Chen liang. Design and Realization of Ship’s Insulation Monitoring System[J].MARINE ELECTRIC \& TECHNOLOGY,2008,28(5):277-280

[2]Chen Shanshan. Research of Online Insulation Monitoring System for the Power System of Ship[J]. SHIP SCIENCE AND TECHNOLOGY,2015,37(1):165-168.

[3]Wu Zhiliang. Ship Power Plant[M]. Dalian: Dalian maritime university press, 2012

[4]Wang Yong. Research of Online Insulation Monitoring System for the Power System of Ship(Master dissertation)[D].Wuhan: Huzhong University of science and techonlogy, 2011.

[5]Qiu Chidong. Programmable Controller and Network Communication[M]. Dalian: Dalian maritime university press, 2011.

[6]Liao Changchu. Programming and Applications of S7-1200 PLC[M]. Beijing: China machine press, 2011.

[7]User Guide of WEINVIEW_EB8000. 2011. 